\title{
Quasiresonant excitation transfer in molecular aggregates ${ }^{a)}$
}

\author{
R. P. Parson and R. Kopelman
}

Department of Chemistry, The University of Michigan, Ann Arbor, Michigan 48109

(Received 27 September 1982; accepted 23 November 1982)

\begin{abstract}
Excitation transfer in finite molecular aggregates is analyzed in the context of the Haken-Strobl model. Explicit solutions are presented for a trimer and a rectangular tetramer. Special emphasis is placed upon population transfer among subunits (monomers, dimers) and its relationship to energy transfer, and upon the problems associated with coherence of this transfer process. These aggregates serve as models for the problem of excitation transfer in disordered media, where partial coherence resulting from short-range interactions has been largely ignored. Our most intriguing result is the greatly diminished effectiveness of the longer-ranged transfer in the presence of short-range clusters. Under some conditions the ensuing energetic mismatches may well dominate the overall energy transport and render invalid the usual description in terms of hopping among individual sites. An application to triplet energy transport in isotopic mixed naphthalene crystals is given; it is seen that the reduced efficiency of non-nearest-neighbor transfer processes reinforces the twodimensional characteristics of the energy transport.
\end{abstract}

\section{INTRODUCTION}

The object of this investigation is to elucidate the nature of energy transfer in collections of molecules coupled by resonant interactions of varying strengths. Special emphasis is devoted to the circumstances under which the aggregate may be described as a collection of smaller aggregates (clusters) weakly coupled to one another. In this context arise such questions as whether the overall dynamics can be described in terms of microscopic transfer rates, whether population transfer among the clusters (which can always be defined) can be identified with energy transfer (which is only well defined in a weak-coupled limit) and whether the population transfer shows oscillatory or overdamped behavior. These closely interrelated questions are usually subsumed under the general topic of "transport coherence." In addition to their intrinsic significance, these questions are relevant to the general problem of excitation transfer in disordered media, since current theoretical models of these processes usually assume incoherent hopping among individual sites, ${ }^{1}$ and do not consider the possible role of partial coherence arising from strong short-ranged interactions.

A popular phenomenological model due to Haken and Strobl $^{2}$ is here applied to two systems: a pair of dimers, and a monomer coupled to a dimer. The limitations of this model have been frequently pointed out ${ }^{3.4}$; it is probably seldom quantitatively applicable to experimentally accessible systems. These defects-neglect of detailed correlations in the phonon-induced fluctuations, neglect of the finite correlation times associated with these fluctuations, and failure to obey the correct detailed-balance requirements at finite temperaturesare for present purposes outweighed by the tremendous simplifications which follow from these model assump tions. These simplifications make it possible to obtain qualitatively useful results by elementary calculations, without necessitating restrictions to weak-coupling cases or the use of poorly controlled approximations.

${ }^{a}$ Supported by NIH Grant No. 2 R01 NS08116-14.
A straightforward application of this model yields results which, while expected from general considerations, do not seem to have been explicitly stated before, at least in this context. Only under very stringent (and not entirely obvious) conditions is it possible to ignore the internal dynamics of the clusters. When an intercluster transfer rate is well defined, it is likely to be smaller than one would predict if one were to ignore the internal dynamics. This result can for the most part be ascribed to energy mismatches between clusters of different types, and leads us to conclude that energy transport in spatially disordered systems dominated by shortrange interactions may be less efficient than one would predict from a hopping model, even at temperatures larger than the strongest interactions in the system. We also find that the distinction between coherent and incoherent intercluster transfer is somewhat ambiguous; this is a typical manifestation of the complicated dynamics of multilevel systems and of few-body problems in general.

\section{SUMMARY OF THE HAKEN-STROBL STOCHASTIC MODEL}

The stochastic description of excitation dynamics pioneered by Haken and Strobl ${ }^{2}$ and extended by others ${ }^{5}$ assumes that the important effects of exciton-vibron and exciton-phonon coupling upon exciton dynamics can be taken into account by considering the matrix elements of the Frenkel Hamiltonian to be random functions of time. Explicitly:

$$
H=\sum_{n} \epsilon_{n}(t) a_{n}^{\dagger} a_{n}+\sum_{n^{\prime} n^{\prime}} J_{n n^{\prime}}(t) a_{n}^{\dagger} a_{n^{\prime}},
$$

where $a_{n}^{\dagger}, a_{n}$ are site creation and annihilation operators, $\epsilon_{n}$ is the local energy of site $n$, and $J_{n n^{\prime}}$ is a resonant coupling between sites $n$ and $n^{\prime}$. The matrix elements $\epsilon_{n}(t)$ and $J_{n n^{\prime}}(t)$ are considered to be Gaussian Markov processes:

$$
\begin{aligned}
& \left\langle\epsilon_{n}(t)\right\rangle=0, \\
& \left\langle J_{n n^{\prime}}(t)\right\rangle \equiv J_{n n^{\prime}}, \\
& \left\langle\epsilon_{n}(t) \epsilon_{n}(0)\right\rangle=\tilde{\gamma}_{n n} \exp \left(-\alpha_{0} t\right),
\end{aligned}
$$




$$
\left\langle J_{n n^{\prime}}(t) J_{n n^{\prime}}(0)\right\rangle=\bar{\gamma}_{n n^{\prime}} \exp \left(-\alpha_{n n^{\prime}} t\right) .
$$

All cross correlations of the form $\left\langle\epsilon_{n}(t) J_{n n^{\prime}}\left(t^{\prime}\right)\right\rangle$ or $\left\langle J_{n n^{\prime}}(t) J_{m m^{\prime}}\left(t^{\prime}\right)\right\rangle$ are ignored. This is done purely for the sake of convenience and has no other justification.

The inverse correlation times $\alpha_{n n^{\prime}}$ provide a measure of the phonon bandwidth. In the original treatment of Haken and Strobl, the limit of very short correlation times (white noise limit) was taken:

$$
\bar{\gamma} e^{-\alpha t} \underset{\substack{\alpha \rightarrow \infty \\ \bar{\gamma} \rightarrow \infty}}{\longrightarrow} \delta(t), \quad \gamma=\frac{\tilde{\gamma}}{\alpha} .
$$

This considerably simplifies the final expressions, although it is not necessary and restricts the applicability of the model. The consequences of retaining a finite correlation time have been investigated ${ }^{5}$; this extension is necessary in order to treat spectral phenomena such as homogeneous Gaussian line shapes, Urbach rule behavior, etc. For present purposes, the greater simplicity of the original Haken-Strobl model justifies its use.

The Hamiltonian prescribed above can be applied to various types of problems. For the study of energy transfer the appropriate procedure is to derive an equation of motion for the thermally averaged density ma$\operatorname{trix} \rho$. To obtain such an expression, one averages the full density matrix $\sigma$ over the stochastic process:

$$
i \frac{d \rho}{d t}=\langle[H, \sigma]\rangle ; \rho=\langle\sigma\rangle \text {. }
$$

This averaging procedure has been carried out by several different methods ${ }^{2,5}$ all of which make use of the Gaussian nature of the process to sum an infinite-order moment expansion by expressing the higher moments in terms of the lower. The most direct of these methods ${ }^{5(b)}$ makes use of the generalized cumulant expansion of $\mathrm{Kubo}^{6}$ to bypass the higher moments entirely. (The operator-ordering problems which frequently vitiate this procedure are absent in the white noise limit, and the exact solution is contained in the first two cumulants. ${ }^{7}$ )

In the Haken-Strobl model, the reduced density matrix equation takes the form

$$
\begin{aligned}
\left(\frac{d \rho}{d t}\right)_{n n^{\prime}}= & -i[\bar{H}, \rho]-\sum_{l}\left(\gamma_{n l}+\gamma_{n^{\prime} l}\right) \rho_{n n^{\prime}}+2 \delta_{n n^{\circ}} \sum_{l} \gamma_{n l} \rho_{l l} \\
& +2\left(1-\delta_{n n^{\prime}}\right) \gamma_{n n^{\prime}} \rho_{n^{\prime} n} ; \bar{H} \equiv\langle H\rangle .
\end{aligned}
$$

This equation falls naturally into three parts. The first term $-i[\bar{H}, \rho]$ has the same form as the full density matrix equation for the entire system. It describes coherent dynamics governed by a thermally averaged Hamiltonian $\vec{H}$. For the case $n=n^{\prime}$, the remaining terms take the form of a master equation:

$$
\left(\frac{\partial \rho_{n n}}{\partial t}\right)_{\text {incoherent }}=2 \sum_{t}\left(\gamma_{n t} \rho_{l l}-\gamma_{l_{n}} \rho_{n n}\right)
$$

with the $\gamma_{n l}$ elements acting as transition rates between sites $n$ and $l$. This describes an incoherent hopping process superposed on the coherent process. Finally, the off-diagonal terms beyond the first describe scattering or dephasing processes:

$$
\left(\frac{\partial \rho_{n n^{\prime}}}{\partial t}\right)_{\text {lncoherest }}=-\sum_{l}\left(\gamma_{n l}+\gamma_{l n^{\prime}}\right) \rho_{n n^{\prime}}+2 \gamma_{n n^{\prime}} \rho_{n^{\prime} n}, \quad\left(n \neq n^{\prime}\right) \text {. }
$$

It may be noted that these terms are local: the incoherent decay of a given off-diagonal element is related only to itself (and to its complex conjugate):

$$
\left(\frac{\partial}{\partial t}\left[\rho_{n n^{\prime}}+\rho_{n^{\prime} n}\right]\right)_{\text {incoherent }}=2\left[\gamma_{n n^{\prime}}-\sum_{l}\left(\gamma_{n t}+\gamma_{l n^{\prime}}\right)\right]\left[\rho_{n n^{\prime}}+\rho_{n^{\prime} n}\right]
$$

The presence of the coherent term in the complete equation destroys this independence and couples the dephasing processes to each other and to the population transfer processes. This is analogous to the exchange-dephasing processes that arise in spin relaxation. ${ }^{8}$

The Haken-Strobl equations form a linear first-order system with constant coefficients, so that their solution is entirely straightforward for any finite aggregate. However, only a few such aggregates, specifically dimers and finite chains, have been studied till now. Two small aggregates are considered here, a rectangular tetramer and a trimer. Both of these lack translational symmetry. The tetramer case is quite simple, and thus will be discussed first; the trimer case, which is much more complicated, will be the concern of the remainder of this work.

\section{THE RECTANGULAR TETRAMER}

The aggregate studied is depicted in Fig. 1. The four sites form two pairs, $(1,2)$ and $(3,4)$, each of which is
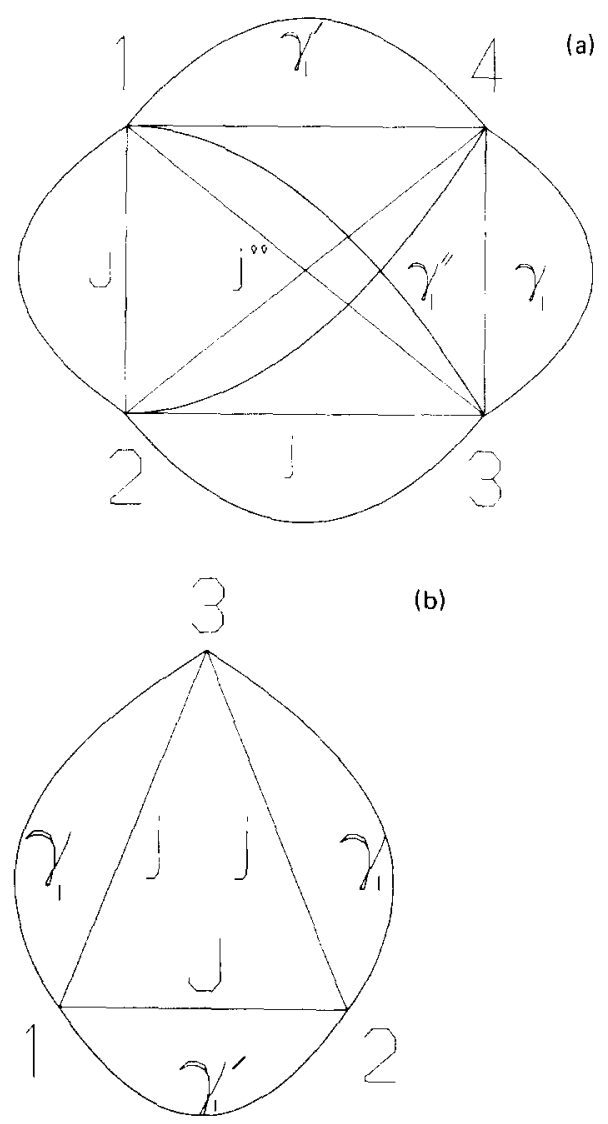

FIG. 1. Parameters for the rectangular tetramer $(A)$ and the trimer $(B)$. Numbers label sites. Straight lines indicate coherent $(J, j)$ interactions, curved lines indicate incoherent $\left(\gamma_{1}\right.$, $\left.\gamma_{1}^{\prime}\right)$ interactions. 
coupled internally by a resonant coupling $J$. The pairs are connected by "weak" coupling elements $j, j$ ". The aggregate is assumed to possess rectangular symmetry. The correlation amplitudes $\gamma_{n n^{\prime}}$ which result from applying the Haken-Strobl model to the aggregate are defined by the figure; the local correlations $\gamma_{n n}$ which are not shown, are referred to as $\gamma_{0}$.

The Haken-Strobl equations for this system can be written:

$$
\frac{d \rho}{d t}=L \rho
$$

in which the Liouville superoperator $L$ is represented by a $16 \times 16$ matrix. The population transfer between the dimers $(1,2)$ and $(3,4)$ is described by the combination:

$$
\left[\begin{array}{cccc}
-2\left(\gamma_{0}+\gamma_{1}+\gamma_{1}^{\prime \prime}\right) & -2 j & -2 j^{\prime \prime} & 0 \\
+2 j & -2\left(\gamma_{0}+\gamma_{1}+\gamma_{1}^{\prime}+2 \gamma_{1}^{\prime \prime}\right) & 0 & +j^{\prime \prime} \\
+2 j^{\prime \prime} & 0 & -2\left(\gamma_{0}+\gamma_{1}^{\prime}+2 \gamma_{1}+\gamma_{1}^{\prime \prime}\right) & +j \\
0 & -4 j^{\prime \prime} & -4 j & -4\left(\gamma_{1}^{\prime}+\gamma_{1}^{\prime \prime}\right)
\end{array}\right] \begin{gathered}
\left(R_{\mathrm{C}}\right) \\
\left(I_{N}\right) \\
\left(I_{T}\right) \\
\left(n_{-}\right)
\end{gathered}
$$

$$
n_{-}=\frac{1}{2}\left[\rho_{11}+\rho_{22}-\rho_{33}-\rho_{44}\right]
$$

This variable (referred to henceforth as the transfer variable) can be obtained as a solution of a $4 \times 4$ problem by defining the following linear combinations:

$$
\begin{aligned}
& I_{T}=\frac{1}{2}\left[\frac{1}{2 i}\left(\rho_{14}-\rho_{41}\right)+\frac{1}{2 i}\left(\rho_{23}-\rho_{32}\right)\right], \\
& R_{C}=\frac{1}{2}\left[\frac{1}{2}\left(\rho_{12}+\rho_{21}\right)-\frac{1}{2}\left(\rho_{34}+\rho_{43}\right)\right], \\
& I_{N}=\frac{1}{2}\left[\frac{1}{2 i}\left(\rho_{13}-\rho_{31}\right)+\frac{1}{2 i}\left(\rho_{24}-\rho_{42}\right)\right] .
\end{aligned}
$$

These combinations are chosen to be purely real and to exploit the spatial symmetry of the system. In this basis the above mentioned $4 \times 4$ problem takes the form:
The important feature to be noted here is that the internal coupling $J$ is entirely absent. This is a direct result of the symmetry of the aggregate and is independent of the relative values of the parameters. The internal dynamics of the dimers does not affect the energy transfer between the dimers.

An explicit solution can easily be obtained for the case $\left(j^{\prime \prime}=0, \gamma_{1}^{\prime \prime}=0\right)$. The transfer variable $n_{-}$is then contained in a $2 \times 2$ submatrix, and the problem becomes isomorphic to that of a dimer. The eigenvalues of the submatrix are:

$$
\lambda_{ \pm}=-\left(\gamma_{0}+4 \gamma_{1}+\gamma_{1}^{\prime}\right) \pm \sqrt{\left(\gamma_{0}+\gamma_{1}^{\prime}\right)^{2}-4 j^{2}} .
$$

These are real for $\left(\gamma_{0}+\gamma_{1}^{\prime}\right) \geq 2 j$, giving rise to overdamped, nonoscillatory transfer, which can be interpreted as "incoherent" dynamics. For the case $j=0$, $\lambda_{-}=-4 \gamma_{1}$, identifying it as the "transfer eigenvalue," that quantity which gives a transfer rate in the weakcoupling limit. For small $j$ one then gets a Golden Ruletype result by expanding the square root:

$$
\lambda_{-} \simeq-4 \gamma_{1}-\frac{2 j^{2}}{\left(\gamma_{0}+\gamma_{1}^{\prime}\right)}
$$

It is interesting to note that the internal nonlocal scattering $\gamma_{i}^{\prime}$ does enter the problem; every scattering event counts in the dephasing processes. Since $\gamma_{1}$ enters to- gether with the local term $\gamma_{0}$, one can think of the combination $\left(\gamma_{0}+\gamma_{1}^{\prime}\right)$ as an effective local scattering (local to the cluster).

For $2 j>\left(\gamma_{0}+\gamma_{1}^{\prime}\right)$, the eigenvalues are complex, and oscillations appear in the transfer variable, giving rise to coherent behavior.

The more general case $j^{\prime \prime}=0$ can be solved numerically, but no new qualitative features are likely to appear so this has not been carried out.

\section{THE TRIMER: FORMULATION AND APPROXIMATE ANALYSIS}

The parameters of the trimer problem are defined analogously to those of the tetramer; see Fig. 1(b). The Liouville matrix is $9 \times 9$; a $4 \times 4$ submatrix for the intercluster transfer can be isolated by taking the combinations:

$$
\begin{aligned}
& n_{-}=\frac{1}{4}\left(2 \rho_{33}-\rho_{11}-\rho_{22}\right), \\
& I_{123}=\frac{1}{2}\left[\frac{1}{2 i}\left(\rho_{13}-\rho_{31}\right)+\frac{1}{2 i}\left(\rho_{23}-\rho_{32}\right)\right], \\
& R_{123}=\frac{1}{2}\left[\frac{1}{2}\left(\rho_{13}+\rho_{31}\right)+\frac{1}{2}\left(\rho_{23}+\rho_{32}\right)\right], \\
& R_{12}=\frac{1}{2}\left[\rho_{12}+\rho_{21}\right] .
\end{aligned}
$$

The submatrix is then:

$$
\left[\begin{array}{cccc}
-2\left(\gamma_{0}+\gamma_{1}\right) & 0 & -j & 0 \\
0 & -\left(2 \gamma_{0}+\gamma_{1}+\gamma_{1}^{\prime}\right) & J & 0 \\
+j & -J & -\left(2 \gamma_{0}+5 \gamma_{1}+\gamma_{1}^{\prime}\right) & -\sqrt{6} j \\
0 & 0 & +\sqrt{6} j & -6 \gamma_{1}
\end{array}\right] \begin{gathered}
\left(R_{12}\right) \\
\left(R_{123}\right) \\
\left(I_{123}\right) \\
\left(n_{-}\right)
\end{gathered}
$$


This matrix displays a complicated series of couplings: $n_{-}$is exchange coupled to a phase variable which is itself exchange coupled to other phase variables (the vacuous term "phase variable" is used here to refer to combinations of off-diagonal elements). Of particular importance is the fact that the intracluster coupling $J$ enters explicitly.

Although a fourth-order secular equation can be solved algebraically, the method is too complicated to be of any practical use. Two approaches are presented here: a simple perturbative calculation in this section and a numerical solution in the next.

In order to use perturbation theory one must specify a small parameter. In keeping with the discussion in the introduction, the dimer coupling $J$ is considered to be large, and the monomer-to-dimer coupling $j$ is treated as the perturbation:

$$
\begin{aligned}
& L=L^{0}+L^{1}, \\
& L^{1}=\left[\begin{array}{cccc}
0 & 0 & -j & 0 \\
0 & 0 & 0 & 0 \\
+j & 0 & 0 & -\sqrt{6} j \\
0 & 0 & +\sqrt{6} j & 0
\end{array}\right] .
\end{aligned}
$$

The first-order correction to the transfer eigenvalue obviously vanishes. The second-order correction is obtained by transforming $L$ to make $L^{0}$ diagonal, which is equivalent to choosing a basis that is diagonal in the isolated dimer eigenstates. (Alternatively, one may express the second-order correction in terms of the right and left eigenvectors of $L$, remembering that $L$ is not Hermitian and that its eigenvectors are therefore not orthogonal). The result is

$\lambda=-\left[6 \gamma_{1}+\frac{6 j^{2}\left(2 \gamma_{0}+\gamma_{1}^{\prime}-5 \gamma_{1}\right)}{\left(2 \gamma_{0}+\gamma_{1}^{\prime}-5 \gamma_{1}\right)\left(2 \gamma_{0}+\gamma_{1}^{\prime}-\gamma_{1}\right)+J^{2}}\right], \quad\left(j>2 \gamma_{1}\right)$.

A similar, but distinct, expression is obtained for the case $j \leq 2 \gamma_{1}$.

The second-order term has a Golden Rule structure, and is in accordance with the concept of a time-independent transition rate between the monomer and dimer states. As expected, the transition rate is diminished by increasing the dimer splitting; this can be ascribed to an "energy-mismatch" effect. Indeed, the scattering parameters and the internal coupling $J$ play analogous roles in this limit, as is apparent from the structure of the expression. Both $J$ and the $\gamma$ parameters tend to retard the transfer. A comparison of the second-order expression to the exact result is given in Fig. 2. One can see that the perturbative treatment works quite well for $j<J$, as expected.

\section{THE TRIMER: NUMERICAL RESULTS}

The principal virtue of the Haken-Strobl model is that it is not restricted to weak-coupling limits, but yields sensible results over the entire range of parameter values. Although the perturbative evaluation in the last section is interesting in that it elucidates the relationship between the exact Haken-Strobl results and concepts

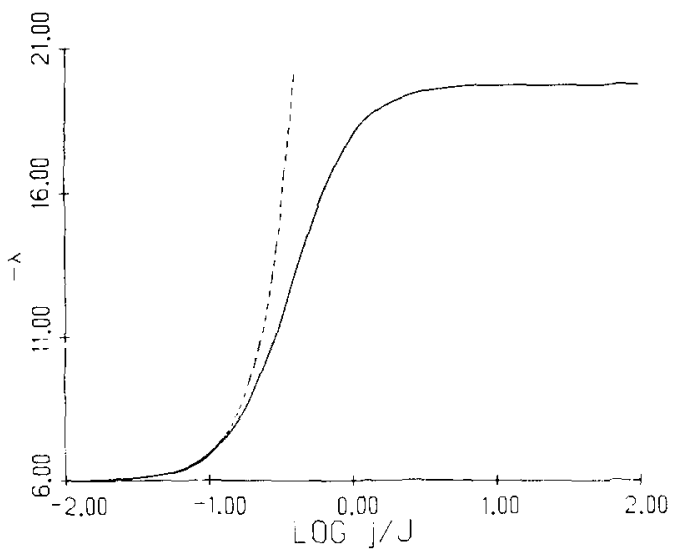

FIG, 2. Comparison of exact (solid line) and approximate dashed line) results for the transfer eigenvalue $\lambda$. In this example, $\gamma_{1}=\gamma_{1}^{\prime}=0.1 \gamma_{0}=0.01 \mathrm{~J} . \lambda$ is measured in units of $\gamma_{1}$.

based upon weak-coupling theory, it does not exploit the full potential of the model. Moreover, within the weak coupling limit one can get more accurate results by using more sophisticated approaches in which the phonon bath is explicitly included. ${ }^{9,10}$ The most interesting feature of the present work arises from a consideration of the numerical results.

The eigenvalues and eigenvectors of the $L$ matrix (by which we mean the $4 \times 4$ submatrix) were obtained by standard methods, using a QR algorithm. The subroutines used were provided by the University of Michigan Computing Center. Since the $L$ matrix contains five independent parameters, some a priori restrictions are called for in order to simplify interpretation. We chose to set $\gamma_{1}=\gamma_{1}^{\prime}$ and $\gamma_{0}=10 \gamma_{1}$, in accordance with the prevalent belief ${ }^{11,12}$ that local scattering dominates. Since we did not study the separate effects of $\gamma_{0}$ and $\gamma_{1}$, we will refer to them in composite as " $\Gamma$." We then followed the behavior of the transfer eigenvalue $\lambda$, and of the eigenvector associated with that eigenvalue, as a function of $j / J$ and $\Gamma / J$.

One rather interesting result that emerges is that there is not a distinct transition from oscillatory to nonoscillatory behavior, such as was seen for the dimer and the rectangular tetramer. The $L$ matrix always has two complex eigenvalues for small $j$ with respect to $J$, independently of $j$. For arbitrarily small $j / J$, an oscillatory component is present in the transfer due to the coupling of the transfer variable to those modes associated with complex eigenvalues. (For $\Gamma$ large with respect to $J$, all eigenvalues are real, but it is natural to ascribe this to a loss of coherence in the internal dynamics of the dimer. The general criterion for this change in the number of eigenvalues has not been obtained explicitly; for $j=0$ it occurs at $J=2 \gamma_{1}$.) One can interpret this phenomenon in various ways, depending upon one's choice of basis. Although, since $n_{-}$is not an observable, one should not ascribe too much significance to the oscillations, they do provide an interesting example of exchange coupling in a three-level system.

Although a sharp transition does not exist, something 


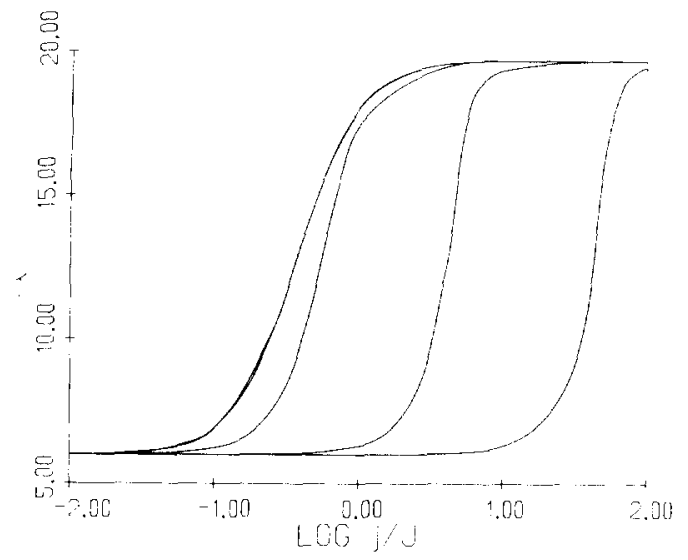

FIG. 3. The transfer eigenvalue $\lambda$ as a function of $j / J$ for 5 values of $\gamma_{0}$ : from left to right, $\gamma_{0}=0.01 J, 0.1 J, J, 10 J$, and $100 \mathrm{~J}$. (The curves for $\gamma_{0}=0.01 \mathrm{~J}$ and $0.1 \mathrm{~J}$ are barely distinguishable.) $\gamma_{1}=\gamma_{1}^{\prime}=0.1 \gamma_{0}$.

very like one can be seen in Figs. 3 and 4 , which show the values obtained for the transfer eigenvalue $\lambda$ and one component of the associated eigenvector, $u$. The component chosen is that which tends to 1 as $j$ tends to 0 ; it thus describes the extent to which the eigenvector is dominated by the transfer variable. The results are represented as functions of $j / J$ for various $\Gamma$. It can be seen that over a short range of $j / J$ (roughly one order of magnitude) the transfer eigenvalue increases rapidly, while the eigenvector component drops from 1 to 0.5 , indicating extensive exchange coupling. One can also see that for $j>J$, the position of the transition is approximately independent of $\Gamma$. The transition can then be interpreted as a quasiresonance effect. An increase in $\Gamma$ serves to sharpen the ons et of the transition slightly, as can be seen by comparing the values of $\lambda$ and $u$ at $j / J=0.1$ in Figs. 3 and 4 . From the same point of view, this can be ascribed to a damping of the quasiresonance by phonon scattering, so that the clusters need to be closer to resonance in order for quasiresonant enhancement to occur. The perturbative expression in the last

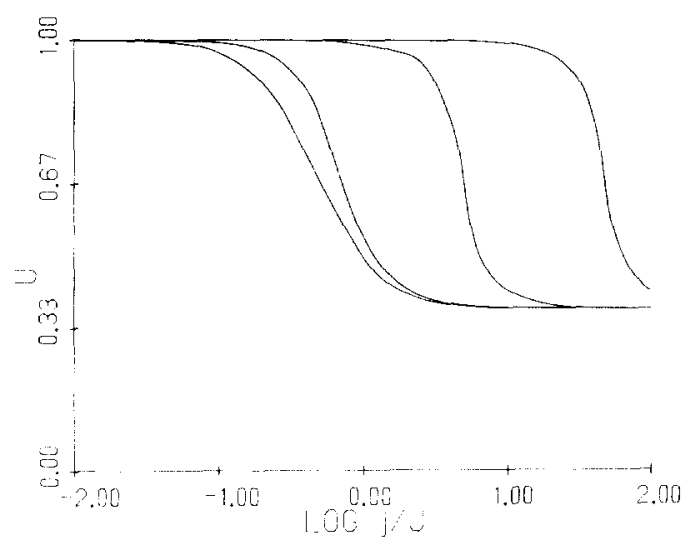

FIG. 4. The "transfer component" $u$ of the eigenvector associated with the transfer eigenvalue as a function of $j / J$ for 4 values of $\gamma_{0}$ : from left to right, $\gamma_{0}=0.1 \mathrm{~J}, J, 10 \mathrm{~J}$, and $100 \mathrm{~J}$. (If it were shown, the curve for $\gamma_{0}=0.01 \mathrm{~J}$ would superimpose upon that for $0.1 J)$. $\gamma_{1}=\gamma_{1}^{\prime}=0.1 \gamma_{0}$.

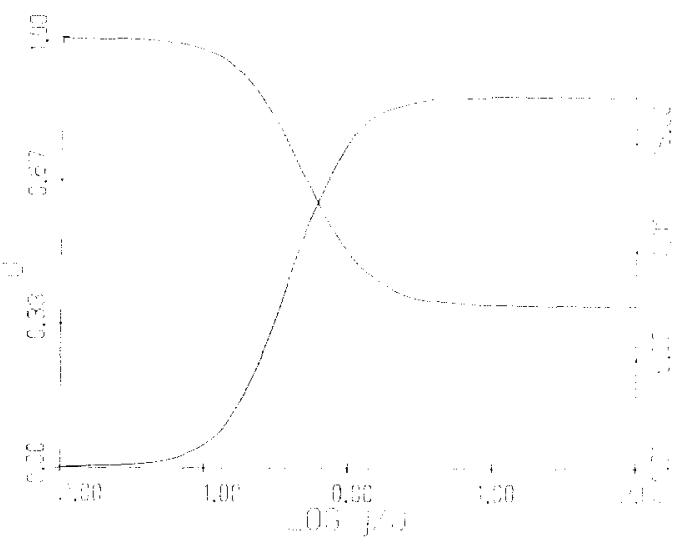

FIG. 5. T'ransfer eigenvalue $\lambda$ and eigenvector component $u$ as functions of $j / J$ for $\gamma_{0}=0.1 J, \gamma_{1} \cdots 0=\gamma_{1}^{\prime} . \quad \lambda$ is measured in units of $0.1 \gamma_{0}$.

chapter also displays this effect, as is expected since it is quite accurate in this region.

For $\gamma_{0}>10 J$ (or $\gamma_{1}>J$ ), the same qualitative features emerge, except for a pronounced shift in the transition region with increasing $\Gamma$. Indeed, under these conditions the transition occurs at a given value of $j / \Gamma$, rather than of $j / J . \quad \Gamma$ has now become the large parameter in the system, and a competition between $j$ and $\Gamma$ dominates the problem. Since all eigenvalues are real, the transfer is necessarily incoherent in this regime of parameter space.

In Fig. 5, the results obtained for $\gamma_{0}<J$ and $\gamma_{1}=0$ are displayed. Since the limiting value of $\lambda$ as $j$ tends to zero is $6 \gamma_{1}$, it is obvious that $\lambda$ must drop to zero in this case. (In order to facilitate comparison to the other figures, $\lambda$ is measured here in units of $0.1 \gamma_{0}$. In the other figures it has units of $\gamma_{1}$, with $\gamma_{0}=10 \gamma_{1}$.) Otherwise, the qualitative form of the results does not differ much from the previous cases. Since the HakenStrobl model neglects cross correlations between local and nonlocal fluctuations, the separate effects of the local and nonlocal parameters may not have much genuine significance.

As a final observation, one may note that although oscillations in the transfer persist below the transition region, their effects are extremely minor since the contributions of the eigenvectors having complex eigenvalues to the transfer variable are very small. This agrees with the purely real form of the second-order result; the oscillations are a higher-order effect. One is reminded of a similar complicated oscillatory motion which appears in the Redfield equations for spin relaxation and which is usually neglected on physical grounds. ${ }^{8(b)}$

\section{DISCUSSION}

Although the calculations presented here are entirely straightforward and the results intuitively reasonable, to our knowledge nothing quite like them has been presented before. Indeed, with few exceptions (notably the interesting finite-chain calculations of Aslangul and 
Kottis ${ }^{13}$ ), the Haken-Strobl model has not been applied to finite systems other than the dimer. This is rather surprising, since it is an extremely useful tool for exploring the qualitative features of relatively complicated systems. Indeed, it may be better suited to this task than to the quantitative interpretation of experimental data, for which is has often been used, since the experimental systems rarely conform to the rather stringent hypotheses of the model. For this reason we have not attempted to calculate such experimental observables as the optical line shape, although this can readily be done (it requires a somewhat different approach, specifically a different subset of density matrix elements).

Since we have not dealt with experimental observables, we have used somewhat imprecise language in several places. For instance, we have referred to a certain combination of diagonal density matrix elements as a "transfer variable," implying that the evolution in time of this quantity reflects the flow of energy in the system. This is only true for weak coupling. For $j=J$, the energy should be regarded as delocalized over the aggregate, and the oscillations in population have little direct significance. We have also suggested, implicitly, an initial condition in which the excitation is completely confined to a portion of the aggregate. This is also only appropriate for weak coupling; it is difficult to conceive of an experiment that would allow such an initial condition when the coupling is strong. Our language, as well as our choice of basis, arises from weak-coupling concepts and is inappropriate outside that regime; this has no bearing on our explicit results, which are of course independent of basis or interpretation. In this context, we remark that the choice of a transfer variable is independent of the basis used for the dimer portion of the aggregate, since all dimer states are summed over; one could also write:

$$
\begin{aligned}
& n_{-}=\frac{1}{4}\left(2 \rho_{33}-\rho_{+4}-\rho_{-.}\right), \\
& | \pm\rangle=\frac{1}{\sqrt{2}}\left(|1\rangle_{ \pm}|2\rangle\right) .
\end{aligned}
$$

The perturbative calculation in Sec. IV involves an implicit transformation to this basis.

With regard to interpretation, perhaps the most relevant quantities are the eigenstates of the $L$ matrix, since they display the extent to which the population changes are coupled to the phase variables, and thus provide an intrinsic measure of the coherence of the aggregate. A complete interpretation, of course, can only be made in the context of a physical observable.

Finally, we point out that the very different behavior of the tetramer and the trimer has a simple physical interpretation. In the former case, there is no mis match between the zero-order $(j=0)$ energies of the two clusters, so that the transfer is resonant. In the latter case, there is such a mismatch, and the transfer is resonant or nonresonant depending upon the relative sizes of $j$ and $J$. This interpretation, while fruitful (and probably valid for a much wider range of circumstances than our model itself is) is not quite general since one can produce cases in which the intracluster interactions enter into the equation of motion for the transfer vari- able even though the clusters are energetically equivalent when the intercluster interaction is neglected. A simple example of this can be obtained by taking the rectangular tetramer in Sec. III and modifying it so that $H_{14} \neq H_{23}$ (so that it becomes a trapezoid). A general discussion of these points is provided in the Appendix.

We now speculate upon the implications of these results for energy transport in disordered media. Our analysis confirms the intuitively reasonable idea that if the intercluster interactions are much weaker than the intracluster ones, then the overall transport of energy in the system may be well described by incoherent hopping among clusters within which the excitation is delocalized. The varying intracluster interaction strengths, arising from the various types of clusters in the medium, produce an effective energetic inhomogeneity in the system. Thus, in a random lattice in which intermolecular interactions vary very strongly with distance (e.g., high-order multipole or exchange interactions) the formation of localized or pseudolocalized cluster states can produce an element of randomness, in addition to the spatial disorder itself, which should be considered when constructing a model for transport in such a system. At low temperatures $(k T<J)$ one may expect additional effects of this general character to arise from detailed-balance and phonon structure effects; these are of course absent in our model, which is restricted to the high temperature limit.

As an example of this situation, we consider the wellstudied first triplet exciton of naphthalene, for which the nearest-neighbor dimer interaction is $1.25 \mathrm{~cm}^{-1} .^{14}$ This is small compared to the optical phonon bandwidth, so that we may expect the Haken-Strobl model to be useful at temperatures considerably greater than $2 J=3.5 \mathrm{~K}$. We take $j$ to be the out-of-plane near-neighbor interaction; this quantity is not known precisely but is known to be less than $0.1 \mathrm{~cm}^{-1} .{ }^{14}$ This particular interaction is very important since it determines the extent to which the excitation migration can be treated as two dimensional. If we take $j=0.03 \mathrm{~cm}^{-1}$, then using data from Refs. 12 and 14 for the parameters $\gamma_{0}=0.11 \mathrm{~cm}^{-1}, \gamma_{1}^{\prime}=0.01$ $\mathrm{cm}^{-1},\left(\gamma_{1}\right.$ is not known but we may neglect it as being at least as much smaller than $j$ as $\gamma_{1}^{\prime}$ is smaller than $J)$ we find from our perturbative expression [Eq. (16)]: $-\lambda=7.7 \times 10^{-4} \mathrm{~cm}^{-1}$, which agrees within experimental accuracy with the result from the numerical calculation: $-\lambda=7.66 \times 10^{-4} \mathrm{~cm}^{-1}$. Thus the transfer from a closely coupled dimer to an out-of-plane monomer is considerably smaller than the transfer rate from a hypothetical noninteracting dimer to the monomer; the latter would be given by the perturbation expression with $J=0: \quad-\lambda=3 j^{2} / \gamma_{0}=2.4 \times 10^{-2} \mathrm{~cm}^{-1}$.

At low temperatures one expects such effects to become more severe. We are presently investigating the incorporation of energetic disorder into models describing transport in spatially disordered systems.

\section{APPENDIX}

We consider here the conditions under which the evolution of the intercluster populations can be de- 
coupled from the intracluster dynamics, i.e., under which the equation of motion of $n_{-}(t)$ does not involve elements of the site-basis Hamiltonian which only couple sites belonging to a single cluster. This discussion is far from complete, but does provide some insight.

It is sufficient to consider only the coherent dynamics, since the incoherent terms connect a nondiagonal density matrix element only to itself and to its complex conjugate. This is a result of a model assumption (absence of cross correlations) whose validity has not been well established; in particular, one may intuitively expect it to fail at low temperatures.

The coherent equations of motion for the real and imaginary parts of the density matrix take the form:

$$
\left.\begin{array}{l}
\frac{d R}{d t}=[H, R] \\
\frac{d I}{d t}=[H, I]
\end{array}\right\}, \quad \rho=R+i I,
$$

where $H$ and $R$ are symmetric, while $I$ is antisymmetric. The equations for the populations are:

$$
\frac{d R_{n n}}{d t}=2(H \cdot I)_{n n}
$$

Partition the set of site indices $S=\{n\}$ into sets corresponding to two clusters: $S=A \cup B$, and write the equation of motion for the difference in populations between the clusters (henceforth, unprimed indices denote members of $A$ and primed indices members of $B$ ):

$$
\begin{aligned}
n_{-}(t)= & \frac{1}{2}\left[\sum_{n} R_{n n^{\prime}}(t)-\sum_{n^{\prime}} R_{n^{\prime} n^{\prime}}(t)\right], \\
\frac{d n_{-}}{d t}= & \sum_{n, t} H_{n l} I_{l n}-\sum_{n^{\prime}, l^{\prime}} H_{n^{\prime} l^{\prime}} I_{l^{\prime} n^{\prime}} \\
& +\sum_{n, l, n^{\prime}, l^{\prime}}\left(H_{n l^{\prime}} I_{l^{\prime} n}-H_{n^{\prime} l} I_{l n}\right) .
\end{aligned}
$$

Since $(H I)$ is antisymmetric, the first two sums, which can be written as traces of $(H I)$ over the two subsets, vanish identically. Equation (A4) then becomes (using the antisymmetry of $I$ ):

$$
\frac{d n_{-}}{d t}=2 \sum_{n n} H_{n n^{\prime}} I_{n^{\prime} n} \text {. }
$$

We now write the equation of motion for $I_{n^{\prime} n}$ :

$\frac{d I_{n^{\prime} n}}{d t}=\sum_{i} H_{n l} R_{l n^{\prime}}-\sum_{l^{\prime}} H_{n^{\prime} l^{\prime}} R_{l^{\prime} n}+\sum_{l, l^{\prime}}\left(H_{n l^{\circ}} R_{l^{\prime} n^{\prime}}-H_{n^{\prime} l} R_{l n}\right)$,

which displays the intracluster elements explicitly. It is clear that in order for the intracluster elements to disappear, some of the $H$ elements must be equal to one another (if one omits the trivial cases of all $H_{n n^{\prime}}$ or all $H_{n l}, H_{n^{\prime}} t^{\prime}$ being equal to zero).

To formulate this point explicitly, we consider sets containing pairs of site indices, i.e., direct product sets:

$$
S^{X} \equiv S \otimes S=(A \otimes A) \cup(B \otimes B) \cup(A \otimes B) \equiv A^{X} \cup B^{X} \cup C^{X} .
$$

Define the following partitions on these sets:

$$
\begin{aligned}
& A^{X}=A^{E_{1}} \cup A^{E_{2}} \cup \cdots \cup A^{E_{n}} \cup A^{N}, \\
& B^{X}=B^{E_{1}} \cup B^{E^{2}} \cup \cdots \cup B^{E_{n}} \cup B^{N} .
\end{aligned}
$$

The sets $A^{E}, B^{E}$ are defined by the property:

$$
\forall(n, m)=A^{E_{i}} \text { and } \forall\left(n^{\prime}, m^{\prime}\right) \in B^{E_{i}}, H_{n m}=H_{n^{\prime} m^{\prime}}=H^{E_{i}} \text {. }
$$

Thus, we pair up equivalent bonds in the separate clus ters. The sets $A^{N}, B^{N}$ contain all those elements which cannot be paired up in this way. The set of intercluster pairs $C$ is also partitioned:

$$
\begin{aligned}
& C^{X}=C^{e_{1}} \cup C^{e^{2}} \cup \cdots \cup C^{e_{n}} \cup C^{N} \\
& \forall\left(n, n^{\prime}\right) \in C^{e_{i}}, \quad H_{n n^{\prime}}=H^{e_{i}},
\end{aligned}
$$

so that equivalent bonds connecting the clusters are collected together.

Now rewrite Eqs. (A5) and (A6):

$$
\frac{d n_{-}}{d t}=2 \sum_{e_{i}} H^{e_{i}} \sum_{n, n^{\prime}}^{\prime} I_{n n^{\prime}}+2 \sum_{\left(n, n^{\prime}\right) \in C^{N}} H_{n n^{\prime}} I_{n^{\prime} n}
$$

$$
\begin{aligned}
\frac{d I_{n^{\prime} n}}{d t} & =\sum_{E_{i}} H^{E i} \sum_{i, l^{\prime}}\left(R_{l n^{\prime}}-R_{l^{\prime} n}\right)+(\text { intercluster terms) } \\
& \sum_{(n, l) \in A N,\left(n^{\prime}, l^{\prime}\right) \in B^{N}}\left(H_{n t} R_{l n^{\prime}}-H_{n^{\prime} t^{\prime}} R_{t^{\prime} n^{n}}\right),
\end{aligned}
$$

where primes on the sums indicate restrictions to members of the set denoted by $e$ or $E$. Then for a given $C$, one can write:

$\frac{d}{d t} \sum_{n, n^{\prime} \in C^{e_{i}}}\left(I_{n n^{\prime}}\right)=\sum_{E_{i}} H^{E_{i}} \sum_{n, n^{\prime}, i, l^{\prime}}^{\prime \prime}\left(R_{l n^{\prime}}-R_{l^{\prime}{ }_{n}}\right)$
$\quad+\left(\right.$ terms in $\left.A^{N}, B^{N}\right)+($ intercluster terms),

where the " indicates the restriction: $H_{n n^{\circ}}=H^{e_{i}}$ and $H_{l_{n}}=H_{l^{\prime} n^{\circ}}=H^{E_{i}}$. The sum runs over all values of the four indices which satisfy these restrictions.

Now clearly, if the combination $\left(R_{l n^{\prime}}-R_{n^{\prime} l}\right)$ appears, then the combination $\left(R_{n l^{\prime}}-R_{n^{\prime} l}\right)$ is also allowed by the restriction. But, since $R$ is symmetric, this term is the negative of the other. The entire sum then vanishes by pairwise cancellation.

Thus, if all intercluster and intracluster bonds can be paired up in this way, i.e., if the residual sets (denoted by superscript $N$ ) are empty, then the contribution of the intracluster bonds to the dynamics of the intercluster transfer disappears. There is no guarantee, however, that these bonds will not reappear when the next set of equations in the hierarchy is written, i.e., the equation of motion of the $R_{l_{n}}, R_{7^{\prime} n^{\prime}}$ which appear in the last sum in Eq. (A6). When the intercluster coupling is small, this truncation of the hierarchy amounts to retaining terms up to second order in the intercluster interaction.

A similar criterion to this can be derived by working in a basis in which the intracluster Hamiltonians are diagonal. The condition on intracluster terms becomes a condition that the clusters have the same energy level structure (when the intercluster terms are set equal to zero) and the condition on intercluster terms becomes a requirement that matrix elements connecting levels in the two clusters which are not isoenergetic should 
vanish. It appears, then, that in general it is not sufficient to assume that the clusters are structurally and environmentally identical in order to guarantee the absence of intracluster terms from the dynamics of the intercluster transfer. One expects, however, that when the intracluster coupling is strong, the effects of the coupling between nondegenerate levels will be much less important than those of couplings between degenerate levels.

The effect of the incoherent terms in the equation of motion is to cause the various phase variables to decay in time. If this damping is very strong for some elements, then modes which are coherently coupled may become effectively decoupled for all but very short times, and the overall dynamics will be simplified.

${ }^{1}$ C. R. Gochanour, H. C. Andersen, and M. D. Fayer, J. Chem. Phys. 70, 4254 (1979); A. Blumen, J. Klafter, and R. Silbey, ibid. 72, 5320 (1980); K. Godzik and J. Jortner, ibid. 72, 4471 (1980).
${ }^{2}$ H. Haken and G. Strobl, Z. Phys. 262, 135 (1973); P. Reineker, in Exciton Dynamics in Molecular Crystals and Aggregates (Springer, Berlin, 1982).

${ }^{3}$ R. Silbey, Annu. Rev. Phys. Chem. 27, 203 (1976).

${ }^{4} R$. Wertheimer and R. Silbey, J. Chem. Phys. 74, 686 (1981).

5 (a) H. Sumi, J. Chem. Phys. 67, 2943 (1977); (b) A. Blumen and R. Silbey, ibid. 69, 3589 (1978); B. Jackson and R. Silbey, ibid. 75, 3293 (1981).

${ }^{6}$ R. Kubo, J. Math. Phys. 4, 174 (1963).

'I. B. Rips and V. Capek, Phys. Status Solidi B 100, 451 (1980).

${ }^{8}$ (a) P. W. Anderson, J. Phys. Soc. Jpn. 9, 316 (1954); (b) C. P. Slichter, Principles of Magnetic Resonance, 2nd ed. (Springer, Berlin, 1980), Chap. 5.

${ }^{9}$ T. F. Soules and C. B. Duke, Phys. Rev. B 3, 262 (1972); S. Rackovsky and R. Silbey, Mol. Phys. 25, 61 (1973).

${ }^{10} \mathrm{~T}$. Holstein, S. Lyo, and R. Orbach, in Laser Spectroscopy of Solids, edited by W. Yen and P. Selzer (Springer, Berlin, 1981), Chap. 2.

${ }^{11}$ V. Ern, A. Suna, Y. Tomkiewicz, P. Avakian, and R. P. Groff, Phys. Rev. B 5, 3222 (1972).

${ }^{12} \mathrm{H}$. Port and H. C. Wolf, Z. Naturforsch. Teil A 30, 1290 (1975).

${ }^{13} \mathrm{C}$. Aslangul and P. Kottis, Adv. Chem. Phys. 41, 321 (1980).

${ }^{14}$ H. Port and D. Rund, J. Mol. Struct. 45, 455 (1978). 